PROCEEDINGS OF THE

AMERICAN MATHEMATICAL SOCIETY

Volume 133, Number 2, Pages 363-367

S 0002-9939(04)07441-6

Article electronically published on September 2, 2004

\title{
NASH EQUIDIMENSIONALITY THEOREM
}

\author{
MASATO FUJITA
}

(Communicated by Michael Stillman)

\begin{abstract}
Consider a Nash mapping of Nash subsets. After a finite number of Nash blowings-up, the Nash mapping induced from it has equidimensional fibers. The purpose of this short note is to show this Nash equidimensionality theorem.
\end{abstract}

\section{INTRODUCTION}

Parusinski proved the following local equidimensionality theorem.

Theorem $1.1(2])$. Let $f: X \rightarrow M$ be a morphism of real analytic spaces, and assume that $M$ is nonsingular. Let $L$ and $K$ be compact subsets of $X$ and $M$, respectively. Then there exist a finite number of analytic morphisms $s_{\alpha}: W_{\alpha} \rightarrow M$ such that

(1) each $s_{\alpha}$ is the composition of a finite sequence of real local blowings-up with smooth nowhere dense centers;

(2) for each $\alpha$ there exists a compact subset $K_{\alpha}$ of $W_{\alpha}$ such that $\bigcup_{\alpha} s_{\alpha}\left(K_{\alpha}\right)=$ $K$;

(3) the strict transforms $\tilde{f}_{\alpha}: \tilde{X}_{\alpha} \rightarrow \tilde{W}_{\alpha}$ of a complexification of $f$ by complexifications of $s_{\alpha}$ satisfy, at every point $x \in \tilde{X}_{\alpha}$ corresponding to $L$, the equidimensionality property:

$$
\operatorname{dim}_{\mathbb{C}}\left(\tilde{f}_{\alpha}^{-1}\left(\tilde{f}_{\alpha}(x)\right) \cap \tilde{X}_{\alpha}^{\prime}, x\right)=\operatorname{dim}_{\mathbb{C}}\left(\tilde{X}_{\alpha}, x\right)-\operatorname{dim}_{\mathbb{R}} M,
$$

for every irreducible component $\tilde{X}_{\alpha}^{\prime}$ of $\tilde{X}_{\alpha}$ at $x$.

We prove the global equidimensionality theorem for any real closed field in the present paper only when $f$ is a Nash mapping of Nash sets. Roughly speaking, our global equidimensionality theorem claims that there exists a composition of a finite sequence of blowings-up whose centers are Nash sets such that the strict transforms of $f$ satisfy the equidimensionality property. We need some definitions to state our result truly. We define Nash blowings-up in Section 2 and describe our result with accuracy in Section 3 ,

Throughout the present paper, $R$ denotes a real closed field.

Received by the editors December 12, 2002 and, in revised form, July 10, 2003.

2000 Mathematics Subject Classification. Primary 14P20.

(C)2004 American Mathematical Society 


\section{NASH BLOWINGS-UP}

A Nash submanifold $M$ of $R^{n}$ is a semialgebraic subset of $R^{n}$ that is simultaneously a $C^{\infty}$ submanifold of $R^{n}$, and a Nash function on $M$ is a semialgebraic $C^{\infty}$ function on $M$. Let $\mathcal{N}(M)$ denote the ring of Nash functions on $M$. Remember that a Nash subset of $M$ is the zero set of a Nash function on $M$. Let $X$ and $Y$ be Nash subsets of Nash manifolds. A $C^{\infty}$ semialgebraic mapping $f: X \rightarrow Y$ is called a Nash mapping. For a Nash subset $X$ of a Nash submanifold $M$ of some Euclidean space, we also call the zero set of a Nash function on $X$ a Nash subset of $X$. Remark that a Nash subset of $X$ is also a Nash subset of $M$ by 1 , Corollary 13, Corollary 15].

We define $\mathcal{N}_{M}^{\text {sa }}$ as the sheaf of Nash functions on $M$ for the semialgebraic topology. Set $\mathcal{N}_{X}^{\text {sa }}:=\mathcal{N}_{M}^{\text {sa }} / \mathcal{I}_{X}$, where $\mathcal{I}_{X}$ denotes the sheaf of ideals of $\mathcal{N}_{M}^{\text {sa }}$ vanishing on $X$.

We will define the blowing-up for a general real closed field.

Definition 2.1. Let $X$ be as above, and let $\mathcal{I}$ be a finite sheaf of ideals of $\mathcal{N}_{X}^{\text {sa }}$ with support $\neq X$. A Nash mapping $\sigma: X^{\prime} \rightarrow X$ is called the Nash blowing-up of $X$ with center $\mathcal{I}$ if the following conditions are satisfied:

(1) $\mathcal{I} \mathcal{N}_{X^{\prime}}^{\text {sa }}$ is invertible;

(2) for any Nash mapping $f: T \rightarrow X$ such that $\mathcal{I N}_{T}^{\text {sa }}$ is invertible, there exists only one Nash mapping $f^{\prime}: T \rightarrow X^{\prime}$ with $\sigma \circ f^{\prime}=f$.

We can define the strict transform of a Nash set by a Nash blowing-up in the same way as Hironaka's definition.

Proposition 2.2. There exists a Nash blowing-up $\sigma: X^{\prime} \rightarrow X$ for any finite sheaf of ideals of $\mathcal{N}_{X}^{s a}$ with support $\neq X$.

Proof. The sheaf $\mathcal{I}$ is generated by $g_{1}, \ldots, g_{m} \in \mathcal{N}(M)$ by [1, Corollary 13, Corollary 15]. Set

$$
\begin{aligned}
Z & =\bigcap_{i=1}^{m} g_{i}^{-1}(0) \cap X \text { and } \\
X^{\prime \prime} & =\left\{(x, s) \in X \times \mathbb{P}^{m-1}(R) ; x \notin Z, g_{i}(x) s_{j}=g_{j}(x) s_{i} \text { for all } i \neq j\right\},
\end{aligned}
$$

where $\mathbb{P}^{m-1}(R)$ denotes the $(m-1)$-dimensional projective space. Define $X^{\prime}$ as the closure of $X^{\prime \prime}$ in $X \times P^{m-1}(R)$ and $\sigma: X^{\prime} \rightarrow X$ as the natural projection. Then $\sigma$ obviously satisfies the first condition of the above definition.

We show that $\sigma$ satisfies the second condition. Let $T \rightarrow X$ be a Nash mapping such that $\left(g_{1} \circ f, \ldots, g_{m} \circ f\right) \mathcal{N}_{T}^{\text {sa }}$ is invertible. We have an open semialgebraic covering $\left\{T_{i}\right\}_{i=1, \ldots, m}$ of $T$ such that $g_{i} \circ f$ generate $\left.\left(g_{1} \circ f, \ldots, g_{m} \circ f\right) \mathcal{N}_{T}^{\mathrm{sa}}\right|_{T_{i}}$. We have only to show the case when $g_{1} \circ f$ generates $\left(g_{1} \circ f, \ldots, g_{m} \circ f\right) \mathcal{N}_{T}^{\text {sa }}$ by the definition of Nash blowings-up. Define $f_{j}^{\prime}$ as the Nash function on $T$ with $g_{j} \circ f=f_{j}^{\prime} g_{1} \circ f$ for all $j \neq 1$. Then the Nash mapping $f^{\prime}: T \rightarrow X^{\prime}$ defined by $f^{\prime}(t)=\left(f(t),\left(1: f_{2}^{\prime}(t): \ldots: f_{n}^{\prime}(t)\right)\right)$ satisfies the equation $\sigma \circ f^{\prime}=f$.

We show the uniqueness of $f^{\prime}$. Let $f^{\prime \prime}: T \rightarrow X^{\prime}$ be another Nash mapping satisfying $\sigma \circ f^{\prime \prime}=f$. Since $g_{i} \circ f$ generates $\left.\left(g_{1} \circ f, \ldots, g_{m} \circ f\right) \mathcal{N}_{T}^{\mathrm{sa}}\right|_{T_{i}}, f^{\prime \prime}\left(T_{i}\right)$ is contained in $X_{i}^{\prime}=\left\{(x, s) \in X^{\prime} ; s_{i} \neq 0\right\}$. It is easy to see that $f^{\prime \prime}(t)$ is determined only by $\sigma \circ f^{\prime \prime}(t)=f(t)$ on each $X_{i}^{\prime}$ by the definition of $X^{\prime}$. We have finished the proof of uniqueness. 
Proposition 2.3. Let $f: X \rightarrow Y$ be Nash mappings between Nash subsets, and let $\mathcal{J}$ be a finite sheaf of ideals of $\mathcal{N}_{Y}^{s a}$. Then there exist a finite sheaf $\mathcal{I}$ of ideals of $\mathcal{N}_{X}^{s a}$ and a Nash mapping $f^{\prime}: X^{\prime} \rightarrow Y^{\prime}$ with $f \circ \sigma_{X}=\sigma_{Y} \circ f^{\prime}$. Here $\sigma_{X}: X^{\prime} \rightarrow X$ and $\sigma_{Y}: Y^{\prime} \rightarrow Y$ denote Nash blowings-up with centers $\mathcal{I}$ and $\mathcal{J}$, respectively.

Proof. By the definition of a Nash subset, a Nash subset $Y$ is the zero set of a Nash function on some Nash manifold $N$. Let $h_{1}, \ldots, h_{m} \in \mathcal{N}(N)$ be generators of $\mathcal{J}$. Define $\mathcal{I}$ as the sheaf of ideals of $\mathcal{N}_{X}^{\text {sa }}$ generated by $h_{1} \circ f, \ldots, h_{m} \circ f$. As shown in the proof of Proposition 2.2, $X^{\prime}$ and $Y^{\prime}$ are subsets of $X \times \mathbb{P}^{m-1}(R)$ and $Y \times \mathbb{P}^{m-1}(R)$, respectively. Consider the restriction of $(f$, id $): X \times \mathbb{P}^{m-1}(R) \rightarrow Y \times \mathbb{P}^{m-1}(R)$ to $X^{\prime}$. It is easy to see that the image $(f$, id $)\left(X^{\prime}\right)$ is contained in $Y^{\prime}$. Hence this restriction $f^{\prime}=\left.(f$, id $)\right|_{X^{\prime}}: X^{\prime} \rightarrow Y^{\prime}$ satisfies the requirement.

\section{NASH EQUIDIMENSIONALITY THEOREM}

The following lemma was proved in $[3$. We will give the proof here for completeness.

Lemma 3.1. Let $Z$ be an algebraic subset of $R^{n}$, and let $p: R^{n} \rightarrow R^{m}$ (resp. $q: R^{n} \rightarrow R^{n-m-1}$ ) be the projection forgetting the last $n-m$ factors (resp. the first $m+1$ factors). Assume $n>m$, and set $k=n-m-1$. Assume furthermore that $Z$ does not have an irreducible component of the form

$$
R^{m+1} \times X .
$$

Then there exist $\tau_{1}, \ldots, \tau_{k} \in R[y]$ such that the polynomial map defined by

$$
\tau(x, y, z)=\left(x, y, z_{1}+\tau_{1}(y), \ldots, z_{k}+\tau_{k}(y)\right)
$$

satisfies that $\left.(p, q) \circ \tau\right|_{Z}$ is a finite-to-one mapping. Here $x=\left(x_{1}, \ldots, x_{m}\right), y$ and $z=\left(z_{1}, \ldots, z_{k}\right)$ denote the coordinate functions of $R^{m}, R$ and $R^{k}$, respectively.

Proof. Let $g \in R[x, y, z]$ be a polynomial function with $g^{-1}(0)=Z$. We choose $\tau_{j}^{\prime} \in R^{k}(j \in \mathbb{N} \cup\{0\})$ satisfying the following condition. For any $j \in \mathbb{N}$, there exists $i>j$ with

(*) $\bigcap_{l=0}^{i}\left\{(x, y, z) \in R^{n} ; g\left(x, l, z-\tau_{l}^{\prime}\right)=0\right\} \neq \bigcap_{l=0}^{j}\left\{(x, y, z) \in R^{n} ; g\left(x, l, z-\tau_{l}^{\prime}\right)=0\right\}$

if the latter set is not empty.

Set $\tau_{0}^{\prime}=0$ first. Choose $i>j$ such that the zero set of $g\left(x^{i}, i, z\right) \in R[z]$ is neither empty nor the entire space $R^{k}$ for some $x^{i} \in R^{m}$. We can in fact choose such a positive integer $i$ and variable $x^{i}$, for otherwise $Z$ must have an irreducible component of the form $R^{m+1} \times X$, which contradicts the assumption. Choose $v^{i}, w^{i} \in R^{k}$ with $g\left(x^{i}, i, v^{i}\right) \neq 0$ and $g\left(x^{i}, l, w^{i}\right)=0$ for all $0 \leq l \leq j$. We have only to set $\tau_{i}^{\prime}=w_{i}-v_{i}$ and choose arbitrary $\tau_{l}^{\prime} \in R^{k}$ for all $j<l<i$. Then the constructed numbers $\tau_{i}^{\prime} \in R^{k}$ satisfy the expected inequality $(*)$.

Let $J \in \mathbb{N}$ satisfy

$$
\bigcap_{l=0}^{J}\left\{(x, y, z) \in R^{n} ; g\left(x, l, z-\tau_{l}^{\prime}\right)=0\right\}=\emptyset .
$$

There exists $\tilde{\tau}=\left(\tau_{1}, \ldots, \tau_{k}\right) \in R[y]^{k}$ with $\tilde{\tau}(l)=\tau_{l}^{\prime}$ for any $0 \leq l \leq J$. 
Set $W=\left\{(x, z) \in R^{n-1} ; \operatorname{dim}(p, q)^{-1}(x, y) \cap \tau(Z)=1\right\}$, where $\tau$ is the mapping defined by

$$
\tau(x, y, z)=\left(x, y, z_{1}+\tau_{1}(y), \ldots, z_{k}+\tau_{k}(y)\right) .
$$

The set $W$ is contained in the intersection

$$
\bigcap_{l=0}^{\infty}\left\{(x, y, z) \in R^{n} ; g\left(x, l, z-\tau_{l}^{\prime}\right)=0\right\}=\emptyset .
$$

Hence $(p, q) \circ \tau$ is finite-to-one.

Lemma 3.2. Let $Z$ be an algebraic subset of $R^{n}$, and let $p: R^{n} \rightarrow R^{m}$ be the projection forgetting the last $n-m$ factors. Set

$$
s=m+\max \left\{\operatorname{dim}\left(p^{-1}(x) \cap Z\right) ; x \in R^{m}\right\} .
$$

Then there exists a Nash mapping $\pi: R^{n} \rightarrow R^{s}$ such that $\left.\pi\right|_{Z}$ is finite-to-one and $p^{\prime} \circ \pi=p$, where $p^{\prime}$ denotes the projection of $R^{s}$ forgetting the last $s-m$ factors.

Proof. We prove this lemma by the induction on $n-s$.

When $s=n$, this lemma is clear.

Assume that $s<n$. Changing the last $n-m$ coordinate functions linearly, we may suppose that $Z$ satisfies the assumption of Lemma 3.1. We can therefore choose the Nash mapping $r: R^{n} \rightarrow R^{n-1}$ such that $p^{\prime \prime} \circ r=p$ and $\left.r\right|_{Z}$ is finite-toone by Lemma 3.1, where $p^{\prime \prime}$ denotes the projection forgetting the last $n-m-1$ factors. The image $r(Z)$ is a semialgebraic set by the Tarski-Seidenberg Principle. Let $Z^{\prime}$ be the Zariski closure of $r(Z)$. Then, by the induction hypothesis, there exists a Nash mapping $\tilde{\pi}: R^{n-1} \rightarrow R^{s}$ such that $\left.\tilde{\pi}\right|_{Z^{\prime}}$ is finite-to-one and $p^{\prime} \circ \tilde{\pi}$ coincides with the projection of $R^{n-1}$ forgetting the last $n-1-m$ factors. The Nash mapping $\pi=\tilde{\pi} \circ r$ satisfies the requirement.

Theorem 3.3. Let $f: X \rightarrow Y$ be a Nash mapping of Nash subsets. Then there exist finite compositions of Nash blowings-up $\sigma_{X}: \tilde{X} \rightarrow X$ and $\sigma_{Y}: \tilde{Y} \rightarrow Y$ such that the induced Nash mapping $\tilde{f}: \tilde{X} \rightarrow \tilde{Y}$ with $\sigma_{Y} \circ \tilde{f}=f \circ \sigma_{X}$ has constant dimensional fibers.

Proof. We first consider that $X$ and $Y$ are contained as semialgebraic sets in Nash submanifolds $M \subset R^{n-m}$ and $N \subset R^{m}$, respectively. Set $u=\min \left\{\operatorname{dim} f^{-1}(y) ; y \in\right.$ $Y\}$ and $v=\max \left\{\operatorname{dim} f^{-1}(y) ; y \in Y\right\}$. We prove the theorem by the induction on $v-u$. We have nothing to prove when $u=v$. Consider the case when $u<v$. Assume that there exists a finite sheaf $\mathcal{J}$ of ideals of $\mathcal{N}_{Y}^{\text {sa }}$ such that the induced Nash mapping $f^{\prime}: X^{\prime} \rightarrow Y^{\prime}$ given in Proposition 2.3 satisfies the inequality $\max \left\{\left(f^{\prime}\right)^{-1}(y) ; y \in Y\right\}<v$. Then the theorem holds true by the induction hypothesis. Hence, we have only to show that such a finite sheaf $\mathcal{J}$ exists.

Consider the Zariski closure $Z$ in $R^{n}$ of the graph of the Nash mapping $f$. Let $p: R^{n} \rightarrow R^{m}$ denote the projection forgetting the last $n-m$ factors. Apply Lemma 3.2 to $Z$ and $p$. There exists a Nash mapping $\pi: R^{n} \rightarrow R^{m+v}$ such that $\left.\pi\right|_{Z}$ is finite-to-one and $p^{\prime} \circ \pi=p$, where $p^{\prime}$ denotes the projection of $R^{m+v}$ forgetting the last $v$ factors. Let $Z^{\prime}$ be the Zariski closure of the semialgebraic set $\pi(Z)$. If the claim is true for $Z^{\prime}$ and $\left.p^{\prime}\right|_{Z^{\prime}}$, then it is also true for $X$ and $f$. Hence we may assume that

- $X \subset R^{n}$ is an algebraic set,

- $Y$ is a Euclidean space, 
- $f$ is the restriction of the projection $p: R^{n} \rightarrow R^{m}$ to $X$ and

- $v=n-m$.

Choose a polynomial function $F \in R\left[x_{1}, \ldots, x_{m}, y_{1}, \ldots, y_{v}\right]$ with $F^{-1}(0)=X$. Consider the expansion

$$
F=\sum_{\left(\alpha_{1}, \ldots, \alpha_{v}\right) \in(\mathbb{N} \cup\{0\})^{v}} a_{\alpha}(x) y^{\alpha},
$$

where $a_{\alpha} \in R\left[x_{1}, \ldots, x_{m}\right]$ and $y^{\alpha}$ denotes the monomial $y_{1}^{\alpha_{1}} \cdots y_{v}^{\alpha_{v}}$. Let $\mathcal{J}$ be the sheaf of ideals of $\mathcal{N}_{Y}^{\text {sa }}$ generated by $a_{\alpha}$. Consider the induced mapping $f^{\prime}$ : $X^{\prime} \rightarrow Y^{\prime}$. Let $\left\{Y_{\alpha}^{\prime}\right\}_{\alpha}$ be a semialgebraic open covering of $Y^{\prime}$ such that $a_{\alpha}$ generates $\mathcal{J N}_{X^{\prime}}^{\mathrm{sa}}$. There exists a Nash function $F_{\alpha}^{\prime} \in \mathcal{N}_{Y}\left(Y_{\alpha}^{\prime}\right)\left[y_{1}, \ldots, y_{v}\right]$ such that $a_{\alpha} F_{\alpha}^{\prime}=$ $F$. We define $p$ as the projection of $R^{n}$ forgetting the last $v$ factors. Especially, $p^{-1}(y) \cap\left(F_{\alpha}^{\prime}\right)^{-1}(0)$ is of dimension $<v$ for all $y \in Y_{\alpha}^{\prime}$. The blow-up $X^{\prime} \cap p^{-1}\left(Y_{\alpha}^{\prime}\right)$ is contained in the zero set $\left(F_{\alpha}^{\prime}\right)^{-1}(0)$ by the definition. We have finished the proof of the claim.

\section{REFERENCES}

[1] M. Coste, M. Ruiz and M. Shiota. Uniform bounds on complexity and transfer of $\mathrm{g}$ lobal properties of Nash functions. J. reine angew. Math., 536:209-235, 2001. MR 2003c:14065

[2] A. Parusinski. Subanalytic functions. Trans. Amer. Math. Soc., 344(2):583-59 5, 1994. MR 94k:32006

[3] M. Shiota. Piecewise linearization of subanalytic functions. II. Real analytic and algebraic geometry (Trento, 1998), 247-307, Lecture Notes in Math., 1420, Springer, Berlin, 1990. MR 91m:32009

Department of Mathematics, Kyoto University, Kyoto, 606-8502 Japan

E-mail address: fujita@math.kyoto-u.ac.jp 